\title{
Fertility Preservation for Young Women with Rectal Cancer-A Combined Approach from One Referral Center
}

\author{
Constantine P Spanos • Apostolos Mamopoulos
}

Received: 13 December 2009/Accepted: 15 June 2010 /Published online: 7 July 2010

(C) 2010 The Society for Surgery of the Alimentary Tract

It was with great interest that we read the publication by Elizur et al. ${ }^{1}$ regarding fertility preservation in young female patients with rectal and anal cancer. Five patients with rectal cancer and one with squamous cancer of the anal canal underwent combined fertility preservation techniques of laparoscopic ovarian transposition, ovarian tissue cryopreservation, and in vitro maturation and vitrification of oocytes and embryos, prior to adjuvant chemoradiation therapy. Of note, the fertility techniques employed were performed at a second operative procedure.

The authors are to be commended for utilizing a multidisciplinary approach in offering a solution to a problem (female fertility and colorectal cancer) for which very little data exist in the literature. However, we have two comments to make.

Firstly, we noted that one rectal cancer patient conceived spontaneously, leading to subsequent childbirth. This demonstrates that ovarian transposition alone was successful. Ovarian transposition can be performed synchronously with primary tumor resection, ${ }^{2}$ thus sparing the patient a second operation. A valid question to the authors would be whether ovarian re-transposition (a third procedure) was required or performed in any of the patients.

Secondly, all patients underwent postoperative chemoradiation. In the current era, neo-adjuvant chemo-

C. P. Spanos $(\bowtie) \cdot$ A. Mamopoulos

Aristotelian University,

Thessaloniki, Greece

e-mail: costasspanos@hotmail.com radiation for advanced rectal cancer is advocated due to the advantage it provides in local control of the disease. $^{3}$ It would be interesting to find out if neoadjuvant chemoradiation was an option for treatment of rectal cancer at the authors' institution, and if the patients in their series had any role in choosing the mode of therapy (adjuvant vs. neoadjuvant).

Young female patients with rectal cancer need to be informed about the effects of the disease and its treatment on fertility. There is very little data regarding the efficacy of current fertility preservation options in female patients with rectal cancer. Moreover, decisions regarding the choice of adjuvant therapy may be affected by the effects of therapy on fertility. ${ }^{4}$ Nevertheless, Elizur et al. have made a very important contribution to the literature, and we await further results of their series of fertility preservation techniques in female rectal cancer patients with anticipation.

\section{References}

1. Elizur SE, Tulandi T, Meterissian S, et al. Fertility preservation for young women with rectal cancer-a combined approach from one referral center. J Gastrointest Surg 2009;13:1111-1115

2. Spanos CP, Mamopoulos A, Tsapas A, et al. Female fertility and colorectal cancer. Int J Colorectal Dis 2008;23:735-743

3. Sauer R, Becker H, Hohenberger W, et al. German Rectal Cancer Study Group. Preoperative versus postoperative chemoradiotherapy for rectal cancer. N Engl J Med 2004;351:17311740

4. Maltaris T, Boehm D, Dittrich R, et al. Reproduction beyond cancer: a message of hope for young women. Gynecol Oncol 2006; 103: 1109-1121 